\title{
Virus Reactivation
}

National Cancer Institute

\section{Source}

National Cancer Institute. Virus Reactivation. NCI Thesaurus. Code C160241.

A mechanism whereby a latent virus that has infected a host cell switches to a lytic stage, undergoing productive viral replication and allowing the virus to spread. 